\title{
Neonatal anemia relates to intestinal injury in preterm infants
}

\author{
Willemien S. Kalteren ${ }^{1 凶}$, Arend F. Bos ${ }^{1}$, Willem van Oeveren ${ }^{2}$, Jan B. F. Hulscher ${ }^{3}$ and Elisabeth M. W. Kooi ${ }^{1}$ \\ (c) The Author(s), under exclusive licence to the International Pediatric Research Foundation, Inc 2021
}

\begin{abstract}
BACKGROUND: Anemia is associated with decreased tissue oxygenation in preterm infants and may contribute to developing necrotizing enterocolitis (NEC). We aimed to investigate whether hemoglobin level is associated with intestinal injury, by comparing anemic infants 10 days prior to red blood cell (RBC) transfusion with non-anemic controls.

METHODS: A nested case-control study in which we matched anemic preterms (gestational age (GA) $<32$ weeks) with non-anemic controls (1:1), based on GA, birth weight (BW), and postnatal age. We measured urinary intestinal fatty acid-binding protein, I-FABP, marker for intestinal injury, twice weekly. Simultaneously, we assessed splanchnic oxygen saturation $\left(\mathrm{r}_{\mathrm{s}} \mathrm{SO}_{2}\right)$ and $\mathrm{r}_{\mathrm{s}} \mathrm{SO}_{2} \mathrm{variability}$. RESULTS: Thirty-six cases and 36 controls were included (median GA 27.6 weeks, BW 1020 grams). Median I-FABP level was higher in cases from 6 days to 24-h before transfusion (median ranging: 4749-8064 pg/ml versus 2194-3751 pg/ml). $\mathrm{R}_{\mathrm{s}} \mathrm{SO}_{2}$ and $\mathrm{r}_{\mathrm{s}} \mathrm{SO}_{2}$ variability were lower in cases than controls shortly before transfusion. Hemoglobin levels correlated negatively with $\mathrm{r}_{\mathrm{s}} \mathrm{SO}_{2}$ and $\mathrm{r}_{\mathrm{s}} \mathrm{SO}_{2}$ variability in cases, and negatively with I-FABP in cases and controls together.

CONCLUSIONS: Urinary I-FABP levels were higher in anemic infants before RBC transfusion than in non-anemic matched controls, suggesting intestinal injury associated with anemia. This may predispose to NEC in some anemic preterm infants.
\end{abstract}

Pediatric Research (2022) 91:1452-1458; https://doi.org/10.1038/s41390-021-01903-x

\section{IMPACT:}

- Anemia is a common comorbidity in preterm infants and may lead to impaired splanchnic oxygen saturation and intestinal tissue hypoxia, a proposed mechanism for NEC.

- Lower hemoglobin level is associated with higher urinary I-FABP levels, a marker for intestinal injury, both in anemic preterm infants and in cases and controls together.

- Lower splanchnic oxygen saturation and reduction of its variability are associated with higher urinary I-FABP levels in anemic preterm infants before their first RBC transfusion.

- These results support the hypothesis that anemia in very preterm infants results in intestinal cell injury, which may precede NEC development in some.

\section{INTRODUCTION}

Anemia occurs frequently in preterm infants during admission to the neonatal intensive care unit (NICU). Multiple endogenous and exogenous factors are involved in anemia development, including decreased iron stores, low erythropoietin levels, infection, and the volume of blood lost to phlebotomy. ${ }^{1,2}$ Hemoglobin $(\mathrm{Hb})$ is essential for organ tissue oxygen delivery, which is driven by cardiac output and oxygen-carrying capacity. Anemia becomes clinically relevant when the oxygen-carrying capacity is too low to meet tissue consumption demands. A red blood cell (RBC) transfusion immediately increases organ oxygen delivery. ${ }^{3,4}$

Recent concerns regarding the association between RBC transfusions and the development of necrotizing enterocolitis (NEC) may have further restricted transfusion policies in preterm infants. ${ }^{5}$ However, anemia itself resulting in tissue hypoxia may also cause intestinal injury, ${ }^{6-8}$ as is also supported by the results of two preclinical studies. ${ }^{9,10}$ It remains unclear, whether this increased risk of NEC is related mainly to the transfusion or to the anemia itself. The role of both clinical risk factors, anemia, and RBC transfusions, has been previously investigated with conflicting results. Several authors reported an association between RBC transfusions and an increased risk of NEC. ${ }^{11-18}$ Others, however, found no association between RBC transfusions and NEC, ${ }^{19-24}$ or even that RBC transfusions are protective. ${ }^{25,26}$

The balance between splanchnic tissue oxygen supply and demand can be assessed using near-infrared spectroscopy (NIRS). ${ }^{27}$ Intestinal fatty acid-binding protein (I-FABP) is a biomarker for the detection and severity of the intestinal injury. ${ }^{28}$ This protein is present in the epithelial cells of the small intestinal tissue and will be immediately released into the circulation after the loss of intestinal wall integrity, and readily excreted by the kidney. ${ }^{28-30}$

In this exploratory study, we aimed to investigate whether anemia is associated with subclinical intestinal injury, by comparing anemic preterm infants from 24-h up to 10 days prior to their first

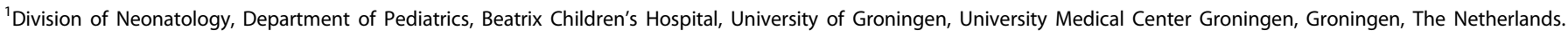

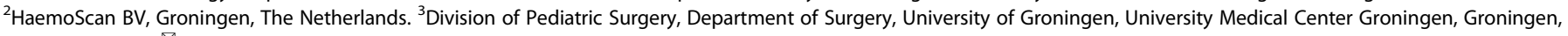
The Netherlands. ${ }^{凶}$ email: w.s.kalteren@umcg.nl
} 
RBC transfusion with non-anemic matched controls. Second, we were interested in whether urinary I-FABP level related to $\mathrm{Hb}$ levels, level of splanchnic tissue oxygen saturation $\left(r_{s} \mathrm{SO}_{2}\right)$, and $\mathrm{r}_{5} \mathrm{SO}_{2}$ variability in all subjects in our study and in cases and controls, separately. We hypothesize to find higher urinary I-FABP levels and lower $\mathrm{r}_{5} \mathrm{SO}_{2}$ in anemic infants compared to non-anemic controls. Furthermore, we hypothesize to find higher I-FABP levels to be associated with lower $\mathrm{Hb}$ and splanchnic oxygen saturation levels.

\section{METHODS}

\section{Study design and study population}

We performed a nested case-control study. Cases and controls were derived from two prospective studies performed at the NICU of the University Medical Center Groningen (UMCG) between July 2018 and December 2020. Between March 2020 and May 2020 no infants were included in the original studies because of the restrictions due to Covid-19. Both trials were registered online in the Dutch Trial Registry (NL6099 and NL6447). In both original studies, we included infants born before 32 weeks of gestation within the first week after birth who did not receive any RBC transfusion before enrollment. Exclusion criteria were an Apgar Score $<5$ at $5 \mathrm{~min}$, intraventricular hemorrhage $>$ Grade II before inclusion, or major congenital or chromosomal abnormalities. Infants of whom parents were unable to understand the Dutch language were also excluded. We obtained written informed parental consent in all cases. The Medical Ethical Review Board of the UMCG approved both original studies.

For the present case-control study, we selected all anemic infants defined as those infants who received an RBC transfusion and matched a control infant to each case, based on gestational age (GA), birth weight (BW), and postnatal age (PNA), with a maximum deviation of $10 \%$. Since we were interested in whether anemia is associated with intestinal injury, we limited data to the samples collected before the moment (PNA) when the first RBC transfusion was administered to the cases. Furthermore, we excluded all samples after confirmed NEC diagnosis ( $\geq$ Bell's Stage II) to prevent measuring NEC-induced intestinal injury. NEC onset was defined as the first abdominal radiograph on which pneumatosis intestinalis, portal venous gas, or both were present. Transfusion thresholds were according to the former Dutch anemia transfusion protocol. ${ }^{31}$ In short, $\mathrm{Hb}$ thresholds were $8.0 \mathrm{mmol} / \mathrm{L}(=12.9 \mathrm{~g} / \mathrm{dL})$ for infants on the first day after birth, and infants on ventilatory support, $7.0 \mathrm{mmol} / \mathrm{L}(=11.3 \mathrm{~g} / \mathrm{dL})$ for infants on other respiratory support, such as CPAP, $6.0 \mathrm{mmol} / \mathrm{L}(=9.7 \mathrm{~g} / \mathrm{dL})$ for infants during the first 4 weeks who were otherwise clinically stable, and 4.5 $\mathrm{mmol} / \mathrm{L}(=7.3 \mathrm{~g} / \mathrm{dL})$ after the first 4 weeks.

\section{Intestinal fatty acid-binding protein}

We collected urine twice weekly during the entire study period. If present for clinical reasons, we collected urine from an indwelling urinary catheter. If not, we placed a small cotton wool in the diaper. Once the cotton was saturated, it was squeezed into a sterile syringe pressing the urine into the urine tube wearing rubber gloves. Next, the urine tubes were frozen at $-80^{\circ} \mathrm{C}$. Batch analysis was performed by a laboratory technician blinded for patient characteristics. To determine the concentration of I-FABP we used sandwich ELISA, based on the capture and biotin-labeled detection antibodies from R\&D Systems (Abingdon, UK). To quantify the amount of I-FABP we used a Streptavidin-HRP and OPD substrate. The standard curve had the highest level of $4000 \mathrm{pg} / \mathrm{mL}$. Samples were diluted two times in $0.1 \%$ BSA/PBS Buffer.

Since we previously reported that the I-FABP to creatinine ratio, to compensate for physiological variations, did not influence I-FABP results in preterm infants and we also demonstrated no additive value of correcting urinary I-FABP levels for creatinine, ${ }^{29}$ we chose not to correct for it.

For statistical analyses, we used a maximum of four urine samples that were collected 10-8 days, 7-5 days, 4-2 days, and 24-h before the PNA at which the first RBC transfusion was administered to cases.

\section{Splanchnic tissue oxygen saturation and extraction}

We measured $\mathrm{r}_{\mathrm{s}} \mathrm{SO}_{2}$ using an INVOS 5100c oximeter and neonatal sensors (Medtronic, Dublin, Ireland). We placed the sensor on the central abdomen under the umbilicus. During the first two weeks after birth, $\mathrm{r}_{5} \mathrm{SO}_{2}$ was measured every day for at least $8 \mathrm{~h}$. Depending on the original study protocol, $\mathrm{r}_{5} \mathrm{SO}_{2}$ was also measured in case of low $\mathrm{Hb}$ levels after these first two weeks. Transcutaneous arterial oxygen saturation $\left(\mathrm{S}_{\mathrm{p}} \mathrm{O}_{2}\right)$ was collected simultaneously. Next, we calculated splanchnic fractional tissue oxygen extraction (sFTOE) by $\left(\left(\mathrm{S}_{\mathrm{p}} \mathrm{O}_{2}-\mathrm{r}_{\mathrm{s}} \mathrm{SO}_{2}\right) / \mathrm{S}_{\mathrm{p}} \mathrm{O}_{2}\right)$. If oxygen consumption is constant, sFTOE represents tissue blood supply. Therefore, it might be a better marker for ischemia than $\mathrm{r}_{5} \mathrm{SO}_{2} \cdot{ }^{32} \mathrm{All} \mathrm{r}_{5} \mathrm{SO}_{2}$ data were collected at 1 $\mathrm{Hz}$ and stored off-line for analysis.

Mean $\mathrm{r}_{5} \mathrm{SO}_{2}$ and sFTOE were calculated during a 2-h period with verified sensor position, for each day of the previously mentioned four time points prior to transfusion or matched PNA. Variability of $\mathrm{r}_{\mathrm{s}} \mathrm{SO}_{2}$ was calculated using the coefficient of variation (CoVar, (SD/mean)*100\%) during 2-h periods for the same four time points. ${ }^{33}$

\section{Clinical variables}

We collected further clinical data from the infants' medical records including Apgar Score at $5 \mathrm{~min}, \mathrm{Hb}$ levels during the study period, presence of a hemodynamic significant patent ductus arteriosus (PDA) requiring treatment, and presence of mechanical ventilation.

\section{Statistical analyses}

We used SPSS Statistics version 24.0 (IBM Corp., Armonk, NY) for statistical analyses. Patient characteristics were described as median, interquartile range (P25-P75, IQR) or $n$, percentage. Non-parametric tests were used to analyze differences between both groups. Next, we determined whether $\mathrm{Hb}$ level, $r_{5} \mathrm{SO}_{2}$, and variability of $\mathrm{r}_{5} \mathrm{SO}_{2}$ were associated with level of I-FABP, and whether $\mathrm{Hb}$ level and $\mathrm{r}_{5} \mathrm{SO}_{2}$ measurements were interrelated, on several time points prior to transfusion using Spearman's Correlation Test in all infants irrespective of group assignment, and in cases and controls separately. Since was suggested that the blood flow in the intestines may be impaired in infants with a PDA, ${ }_{1}^{34,35}$ we additionally calculated partial correlation coefficients to correct for the potential influence of a PDA. Finally, we performed linear regression analyses to determine the effect size of $\mathrm{Hb}$ level, $\mathrm{r}_{5} \mathrm{SO}_{2}$, and $\mathrm{r}_{5} \mathrm{SO}_{2}$ variability on urinary I-FABP level closest to RBC transfusion. Factors associated with $p<0.10$ were included in the multivariable model. Throughout all analyses $p<0.05$ was considered statistically significant.

\section{RESULTS}

\section{Patient characteristics}

The final cohort of enrolled infants in the two original prospective studies consisted of 140 preterm infants (Fig. 1). Forty infants received an $\mathrm{RBC}$ transfusion at a median of 10 days after birth. We were able to select controls from the remaining 100 infants for 36 cases, resulting in a total of 72 included infants in the current study. Of all potential data collected in cases and controls up to the PNA of the first RBC transfusion, we missed one (1\%) urinary sample and four (3\%) $\mathrm{r}_{\mathrm{s}} \mathrm{SO}_{2}$ measurements in cases, and one (1\%) urinary sample and nine $(7 \%) \mathrm{r}_{\mathrm{s}} \mathrm{SO}_{2}$ measurements in controls, due to logistic reasons.

No differences in baseline characteristics were present between cases and controls, except for the higher prevalence of mechanical ventilation and a confirmed PDA in cases (Table 1). In cases, median postnatal age of RBC transfusion was Day 11 . Controls had a similar postnatal age of 11 days (IQR 8-18). Regarding advancing feeding, we observed no differences between cases and controls. Four cases experienced a short period of gastroesophageal reflux, abdominal distension, and/or feeding difficulties, whereas feeding was temporarily halted in three controls. Hemoglobin levels were lower in cases already from 7 to 5 days prior to transfusion (Fig. 2a).

\section{Intestinal fatty acid-binding protein}

In Fig. 2b, we present the course of I-FABP up to 10 days prior to RBC transfusion. Median levels of I-FABP were higher in cases than in controls on subsequent time points from seven to five days until 24-h before RBC transfusion (Fig. 2b).

Splanchnic tissue oxygen saturation and extraction, and $\mathrm{r}_{5} \mathrm{SO}_{2}$ variability

At time point 24-h before RBC transfusion, cases had lower $\mathrm{r}_{5} \mathrm{SO}_{2}$ than controls (Fig. 2c). Taken all infants together, $\mathrm{Hb}$ level correlated with $\mathrm{r}_{\mathrm{s}} \mathrm{SO}_{2}$ 24-h before transfusion (Spearman's $\rho=$ $0.492, p<0.01$ ). 


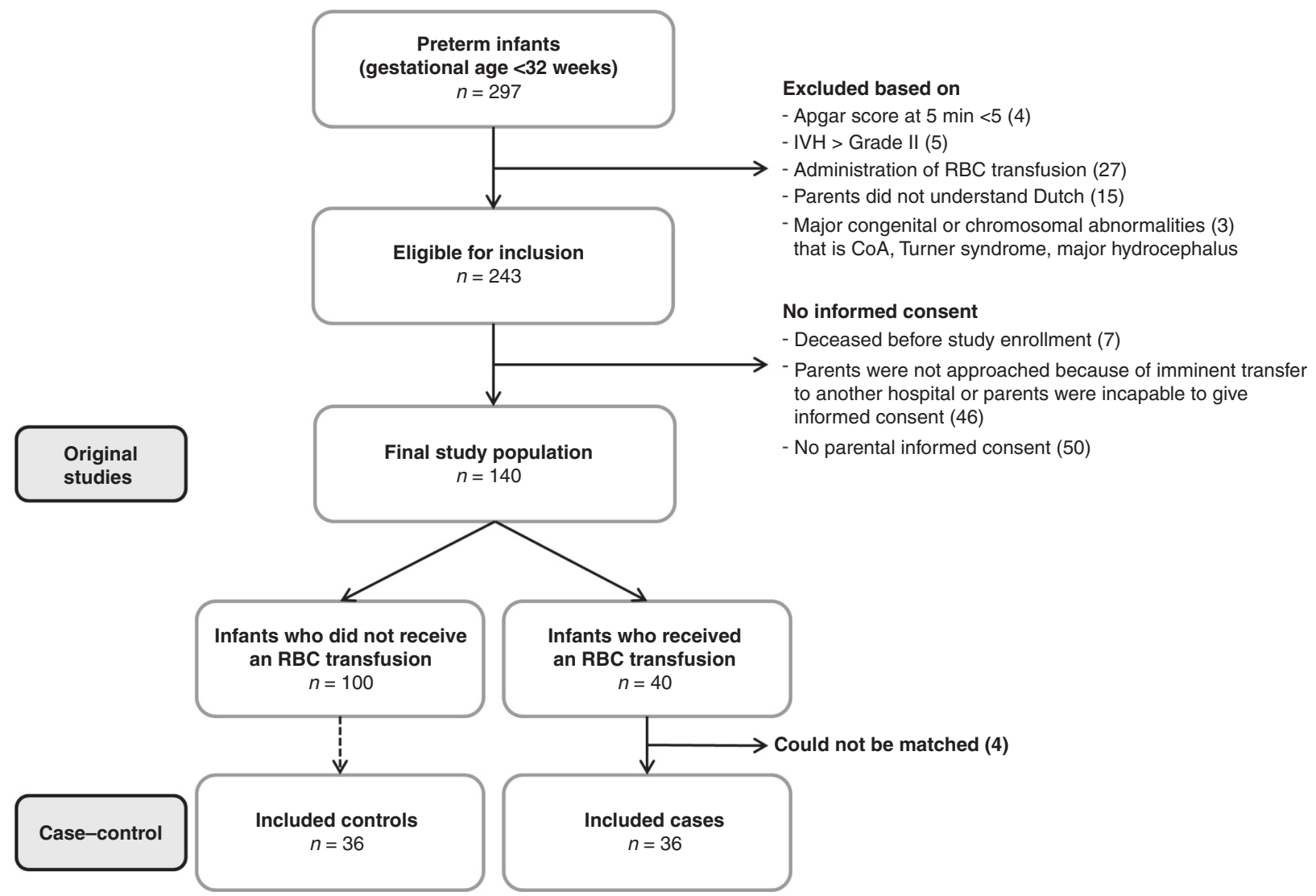

Fig. 1 Flow of participants. IVH intraventricular hemorrhage; RBC red blood cell, CoA coarctation of the aorta, NICU neonatal intensive care unit.

Table 1. Patients' baseline characteristics.

\begin{tabular}{|c|c|c|}
\hline $\begin{array}{l}\text { Cases } \\
n=36\end{array}$ & $\begin{array}{l}\text { Controls } \\
n=36\end{array}$ & $p$ \\
\hline $27.6(26.6-28.6)$ & $27.6(27.3-28.9)$ & 0.29 \\
\hline 985 (850-1204) & $1047(950-1187)$ & 0.24 \\
\hline $11(31)$ & $11(31)$ & $>0.99$ \\
\hline $16(44)$ & $20(56)$ & 0.35 \\
\hline $25(69)$ & $23(64)$ & 0.62 \\
\hline $17(47)$ & $17(47)$ & $>0.99$ \\
\hline $8(6-8)$ & $8(7-8)$ & 0.35 \\
\hline $21(58)$ & $25(69)$ & 0.33 \\
\hline $10.1(8.9-11.4)$ & $10.6(9.7-11.9)$ & 0.11 \\
\hline $21(58)$ & $11(31)$ & 0.02 \\
\hline $17(47)$ & $4(11)$ & $<0.01$ \\
\hline $11(8-18)$ & $11(8-18)$ & 0.98 \\
\hline
\end{tabular}

PNA on day of RBC transfusion ${ }^{d}$

$11(8-18)$

0.98

Displayed as median, $\mathrm{P} 25-\mathrm{P} 75$, or as $n$, percentage when appropriate.

$S G A$ small-for-gestational age (<10th percentile), Hb hemoglobin, PDA patent ductus arteriosus, PNA postnatal age, $R B C$ red blood cell.

${ }^{\mathrm{a}} \mathrm{A}$ full course is defined as delivery at least $24-\mathrm{h}$ after the second dose.

${ }^{\mathrm{b}}$ Mechanical ventilation indicates synchronized intermittent positive pressure ventilation, synchronized intermittent mechanical ventilation, or high-frequency oscillatory ventilation.

'PDA indicates a hemodynamically significant left-to-right shunt that, according to the team of neonatologists and cardiologists, required treatment, i.e., a symptomatic PDA.

${ }^{d}$ Control infants did not receive an RBC transfusion, but were matched on PNA. 

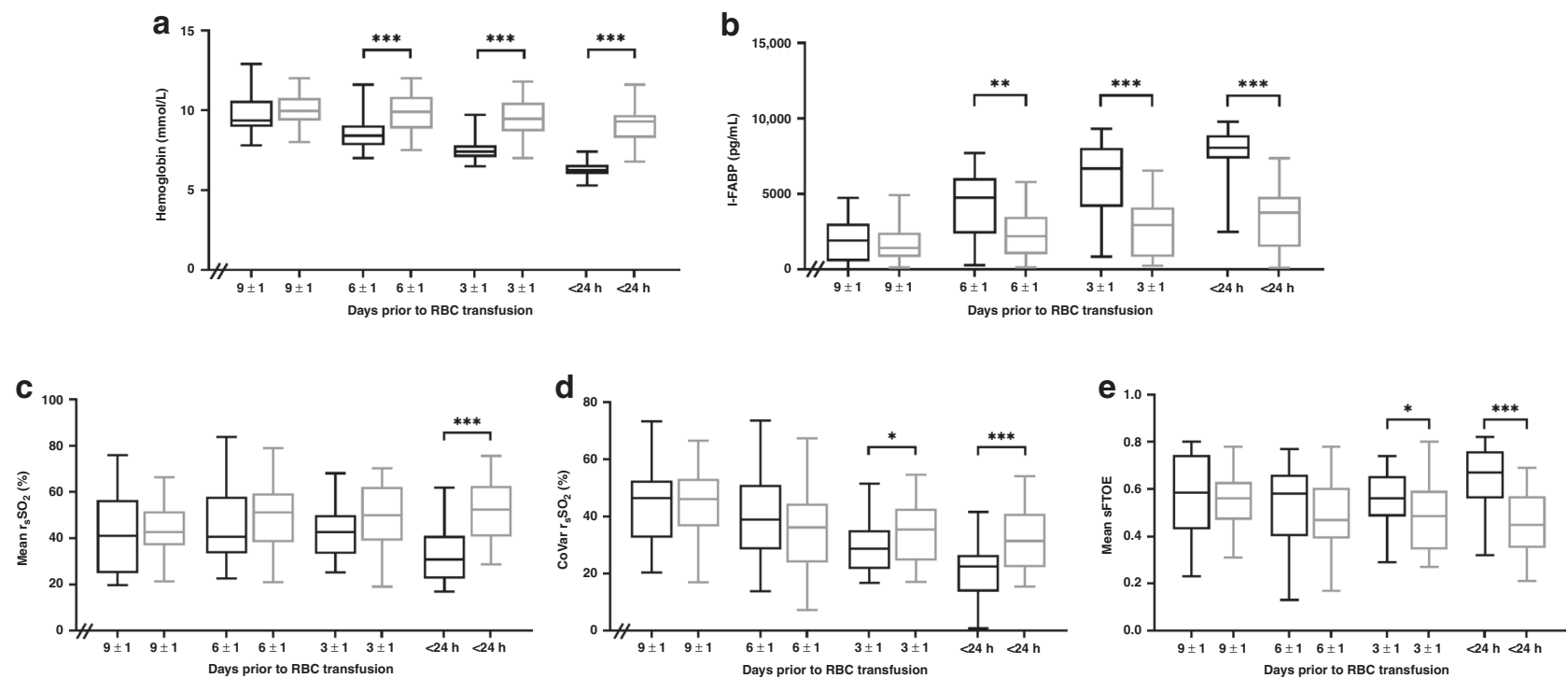

Fig. 2 Course of hemoglobin level, urinary I-FABP, splanchnic oxygen saturation and extraction, and variability of splanchnic oxygen saturation in cases and controls on the same postnatal day. a Course of hemoglobin level. b Course of urinary I-FABP. C Course of mean $\mathrm{r}_{\mathrm{s}} \mathrm{SO}_{2}$. d Course of CoVar $\mathrm{r}_{\mathrm{s}} \mathrm{SO}_{2}$. e Course of mean cFTOE. Data are presented in box-and-whisker plots; black boxes represent cases; gray boxes represent controls; ${ }^{*} p<0.05 ;{ }^{* *} p<0.01 ;{ }^{* * *} p<0.001$. I-FABP intestinal fatty acid-binding protein, $\mathrm{RBC}$ red blood cell, $\mathrm{r}_{\mathrm{s}} \mathrm{SO} \mathrm{O}_{2}$ splanchnic tissue oxygen saturation, CoVar coefficient of variation, sFTOE splanchnic fractional tissue oxygen extraction.

In Fig. 2d, we present a wide range in variability of $r_{s} \mathrm{SO}_{2}$, especially 10-8 days and 7-5 days prior to transfusion. On both 4-2 days before and 24-h before RBC transfusion, cases had a lower variability of $\mathrm{r}_{\mathrm{s}} \mathrm{SO}_{2}$ than controls (Fig. $2 \mathrm{~d}$ ). Taken all infants together, $\mathrm{Hb}$ levels 24-h before RBC transfusion correlated with the concomitant $\mathrm{r}_{\mathrm{s}} \mathrm{SO}_{2}$ variability (Spearman's $\rho=0.402, p<0.01$ ).

Figure $2 \mathrm{e}$ shows that cases had higher sFTOE than controls on both 4-2 days before and 24-h before RBC transfusion.

\section{Association between hemoglobin, splanchnic oxygen saturation, and I-FABP}

In all infants, levels of urinary I-FABP correlated negatively with $\mathrm{Hb}$ levels on subsequent time points from 7 to 5 days until 24-h before RBC transfusion (Table 2A). Levels of urinary I-FABP also correlated negatively with $\mathrm{r}_{\mathrm{s}} \mathrm{SO}_{2}$ 24-h before transfusion, and with $\mathrm{r}_{\mathrm{s}} \mathrm{SO}_{2}$ variability both 4-2 days and 24-h before RBC transfusion. After adjustment for the presence of a PDA (Table 2B), or mechanical ventilation at the time of the measurements (Table $2 \mathrm{C}$ ), similar results were found.

When we repeated the analyses in cases and controls separately, negative correlation coefficients between $\mathrm{Hb}$ level and I-FABP remained significant in cases and disappeared in controls (Table 3 ). In both cases and controls, lower $\mathrm{r}_{5} \mathrm{SO}_{2}$ variability $24-h$ before transfusion and on matched postnatal age, correlated with higher I-FABP levels.

In Table 4, we present the results of the linear regression analyses on the level of urinary I-FABP 24-h before RBC transfusion in all infants. Both lower concomitant $\mathrm{Hb}$ level, $\mathrm{r}_{\mathrm{s}} \mathrm{SO}_{2}$, and $\mathrm{r}_{\mathrm{s}} \mathrm{SO}_{2}$ variability were associated with higher I-FABP level. The multivariable model revealed that lower $\mathrm{Hb}$ level and lower $\mathrm{r}_{5} \mathrm{SO}_{2}$ variability remained associated with higher I-FABP level $<24-\mathrm{h}$ before RBC transfusion.

\section{DISCUSSION}

This study demonstrated that subclinical intestinal injury, as measured by urinary I-FABP levels, was present in anemic preterm infants from 6 days up to 24-h before their first RBC transfusion. Splanchnic oxygen saturation was lower in cases compared to controls 24-h before transfusion. The $\mathrm{r}_{\mathrm{s}} \mathrm{SO}_{2}$ variability was lower in cases than controls already from 3 days before RBC transfusion.
Furthermore, we found that lower hemoglobin level was associated with higher urinary I-FABP from 6 days prior to RBC transfusion. Lower splanchnic oxygen saturation and its variability were also associated with higher urinary I-FABP 24-h before transfusion. These associations were irrespective of the presence of a PDA or mechanical ventilation. Finally, we demonstrated that lower $\mathrm{Hb}$ level from 6 days up to 24-h before RBC transfusion remained associated with higher I-FABP level in cases, but not in controls. Separately in both cases and controls, lower variability of $\mathrm{r}_{\mathrm{s}} \mathrm{SO}_{2}$ remained associated with higher I-FABP level 24-h before transfusion.

As hypothesized, I-FABP levels were higher in cases who ultimately received an $\mathrm{RBC}$ transfusion than in matched controls. Primarily, I-FABP is expressed by epithelial cells on the crypts and the villi, which are known to be the initial destruction site in various intestinal diseases. ${ }^{36}$ Cases showed higher I-FABP levels already from 6 days before transfusion, when $\mathrm{Hb}$ levels were already lower than in controls. Elevated I-FABP is associated with both severe intestinal injury, e.g., intestinal ischemia or NEC, and relatively mild injury, such as a viral gastroenteritis, resulting in the loss of intestinal wall integrity. ${ }^{37}$ Our results suggest that the elevated levels of I-FABP that we found, represent subclinical intestinal injury in anemic preterm infants, based on the relatively low levels of urinary I-FABP compared to I-FABP levels that were previously published at the time of NEC onset. ${ }^{29,38}$ We speculate that a mild intestinal injury may be well tolerated in the stable infants without any large additional risks. However, in anemic infants with, for example, a different fetal condition as reflected by either an NEC-associated gut microbiota ${ }^{39}$ or an already compromised cerebral oxygen saturation on the first days after birth, ${ }^{40}$ the anemia-related intestinal injury may become clinically significant and possibly precede the onset of NEC.

During the week preceding the transfusion up until 24-h pretransfusion, we were unable to show a difference in splanchnic oxygen saturation between cases and control infants, regardless of differences in $\mathrm{Hb}$ level. $\mathrm{R}_{\mathrm{s}} \mathrm{SO}_{2}$ values showed a wide variation between infants, as also has been reported in previous research. ${ }^{27,41}$ This may be caused by the fact that $r_{s} \mathrm{SO}_{2}$, mainly reflecting venous blood, represents the constantly changing balance between oxygen supply and demand. Possibly, we were unable to demonstrate lower $\mathrm{r}_{\mathrm{s}} \mathrm{SO}_{2}$ or higher sFTOE in anemic infants because of an already decreased intestinal metabolism and 
Table 2. Correlation coefficients between hemoglobin level, splanchnic oxygen saturation, and urinary I-FABPs at several time points prior to red blood cell transfusion.

\section{I-FABP, pg/ml}

$$
9 \pm 1 \text { days }
$$$$
6 \pm 1 \text { days }
$$

0.134

$-0.570^{* * *}$
-0.028
0.046

$-0.529 * * *$

$-0.179$

$-0.189$

0.156
$-0.009$

$-0.035$ $-0.272^{\triangle}$

$-0.176$

$$
-0.203
$$

$3 \pm 1$ days

$<24-h$

$-0.773^{* * *}$
-0.068

$-0.196^{\Delta}$

$-0.706 * * *$

$-0.016$

$-0.202^{\Delta}$

$-0.317^{* * *}$

$-0.590^{* * * *}$

(C) Partial correlation coefficients adjusted for mechanical ventilation at time of measurement ${ }^{\mathrm{b}}$

$\mathrm{Hb}$ level, $\mathrm{mmol} / \mathrm{L}$

$\mathrm{R}_{5} \mathrm{SO}_{2}$ (\%)

$-0.562^{* * *}$

$-0.114$

$-0.002$

$-0.733^{* * *}$
-0.062
$-0.221^{\Delta}$

$-0.739^{* * *}$

$-0.258^{*}$

$-0.556^{* * *}$

Displayed as Spearman's $\rho .{ }^{\Delta}$ indicates $p<0.1 ;{ }^{*} p<0.05 ;{ }^{* *} p<0.01 ;{ }^{* * *} p<0.001$

I-FABP intestinal fatty acid-binding protein, $r_{s} \mathrm{SO}_{2}$ splanchnic tissue oxygen saturation, CoVar coefficient of variation, $P D A$ patent ductus arteriosus.

aPDA indicates a hemodynamically significant left-to-right shunt that, according to the team of neonatologists and cardiologists, required treatment, i.e., a symptomatic PDA.

${ }^{b}$ Mechanical ventilation indicates synchronized intermittent positive pressure ventilation, synchronized intermittent mechanical ventilation, or high-frequency oscillation.

Table 3. Correlation coefficients between hemoglobin level, splanchnic oxygen saturation, and urinary I-FABPs at several time points prior to red blood cell transfusion in cases and controls, separately.

\section{I-FABP, pg/ml}

$$
9 \pm 1 \text { days }
$$

$$
6 \pm 1 \text { days }
$$

$3 \pm 1$ days $<24-h$

\section{(A) Cases}

$\mathrm{Hb}$
level, $\mathrm{mmol} / \mathrm{L}$ $-0.315$ $-0.601^{* * *}$ $-0.778^{* * *}$ $-0.358^{*}$ $\mathrm{R}_{\mathrm{s}} \mathrm{SO}_{2}$ (\%) $-0.420^{*}$ $-0.021$

0.150 $-0.104$ 0.078 $-0.042$

$\mathrm{CoVar}$ $\mathrm{r}_{\mathrm{s}} \mathrm{SO}_{2}(\%)$

(B) Controls

$\begin{array}{lrrrc}\mathrm{Hb} & -0.013 & -0.244 & -0.300^{\Delta} & -0.193 \\ \text { level, mmol/L } & & & & \\ \mathrm{R}_{5} \mathrm{SO}_{2}(\%) & 0.103 & -0.009 & 0.201 & 0.205 \\ \mathrm{CoVar} & 0.222 & 0.093 & -0.029 & -0.348^{*} \\ \mathrm{r}_{\mathrm{s}} \mathrm{SO}_{2}(\%) & & & & \end{array}$

Displayed as Spearman's $\rho .{ }^{\Delta}$ indicates $p<0.1 ;{ }^{*} p<0.05 ;{ }^{* * *} p<0.001$. I-FABP intestinal fatty acid-binding protein, $\mathrm{Hb}$ hemoglobin, $r_{5} \mathrm{SO}_{2}$ splanchnic tissue oxygen saturation, CoVar coefficient of variation.

concomitant decreased intestinal oxygen extraction, resulting in relatively high $\mathrm{r}_{\mathrm{s}} \mathrm{SO}_{2}$ levels in anemic infants. This, however, is a highly speculative explanation. During the last 24-h before the transfusion, however, we did find lower $\mathrm{r}_{\mathrm{s}} \mathrm{SO}_{2}$ in cases than in controls. This may reflect intestinal hypoxia during progressive anemia, potentially aggravated by a reduced blood flow to the intestines due to redistribution of blood to the vital organs, ${ }^{6}$ as was previously demonstrated in sheep. ${ }^{42}$ During this situation of limited splanchnic oxygen saturation, the oxygen demand of the intestinal mucosa, for example, related to feedings, may not be met as a result of impaired oxygen delivery to the intestines during severe anemia. ${ }^{43}$ This may also contribute to the developing intestinal injury we found in anemic infants.
Regarding the variability of $\mathrm{r}_{\mathrm{s}} \mathrm{SO}_{2}$ as reflected by its CoVar, we found a lower $\mathrm{r}_{\mathrm{s}} \mathrm{SO}_{2}$ variability in cases than in controls from 3 days before RBC transfusion. The diminished $\mathrm{r}_{\mathrm{s}} \mathrm{SO}_{2}$ variability might represent a reduced capacity and function of the splanchnic vasculature, similar to decreased heart rate variability that reflects the reduced ability to adapt to changes in the activity of the autonomic nervous system, in case of imminent sepsis in preterm infants. ${ }^{44,45}$ Possibly, the degree of $\mathrm{r}_{\mathrm{s}} \mathrm{SO}_{2}$ variability may provide a more accurate reflection of splanchnic oxygenation, rather than actual $\mathrm{r}_{\mathrm{s}} \mathrm{SO}_{2}$ values. Thus, reduced $\mathrm{r}_{\mathrm{s}} \mathrm{SO}_{2}$ variability may be a marker for intestinal hypoxia. Another explanation is that a reduced $\mathrm{r}_{\mathrm{s}} \mathrm{SO}_{2}$ variability indicates hypoxic intestinal injury, as lower $\mathrm{r}_{\mathrm{s}} \mathrm{SO}_{2}$ variability was associated with higher levels of I-FABP. In this sense, a reduced $\mathrm{r}_{\mathrm{s}} \mathrm{SO}_{2}$ variability may serve as a marker for intestinal injury. Reduction of splanchnic variability has previously also been shown as an early marker for NEC development in preterm infants. ${ }^{46-49}$ The fact that the association between lower $\mathrm{r}_{\mathrm{s}} \mathrm{SO}_{2}$ variability and higher I-FABP levels remained significant in non-anemic controls, in contrast to the association between $\mathrm{Hb}$ level and I-FABP, may support the speculation that a reduced $\mathrm{r}_{\mathrm{S}} \mathrm{SO}_{2}$ variability reflects intestinal injury.

Remarkably, we found that $\mathrm{Hb}$ levels were lower and I-FABP levels higher in cases than controls already from 6 days before RBC transfusion. Variability of $\mathrm{r}_{5} \mathrm{SO}_{2}$ was lower from 3 days before the transfusion, whereas actual $\mathrm{r}_{5} \mathrm{SO}_{2}$ only differed 24-h before RBC transfusion between cases and controls. This timeline is intriguing and may suggest that imminent anemia plays an important role in the pathogenesis of intestinal cell injury. Based on these subsequent events, we speculate that a decreased oxygen-carrying capacity may induce intestinal injury. A reduced $\mathrm{r}_{\mathrm{s}} \mathrm{SO}_{2}$ variability may either reflect already existing intestinal injury, be it less pronounced and less sensitive than that reflected by I$\mathrm{FABP}$, or it represents the preceding intestinal hypoxia, which is seen in lower $\mathrm{r}_{\mathrm{s}} \mathrm{SO}_{2}$ only 24 -h before transfusion. The increase in urinary I-FABP, however, is considerably lower compared to infants with confirmed NEC. ${ }^{29,38}$ This may support the notion that anemia-related intestinal injury starts as a relatively mild injury, which may further develop into more severe injury depending on other clinical risk factors for intestinal injury. Despite the difference 
Table 4. Associations between hemoglobin level, splanchnic oxygen saturation, and urinary I-FABP level 24-h before red blood cell transfusion, as analyzed by linear regression analyses.

\begin{tabular}{|c|c|c|c|c|c|c|c|c|}
\hline & \multicolumn{4}{|c|}{ Univariate analyses on I-FAPB $<24 \mathrm{~h}$ before $\mathrm{RBCT}$} & \multicolumn{4}{|c|}{ Multivariable model on I-FAPB $<24 \mathrm{~h}$ before $\mathrm{RBCT}^{\mathrm{a}}$} \\
\hline & $B(95 \% \mathrm{Cl})$ & Beta & $R^{2}$ & $p$ & B $(95 \% \mathrm{Cl})$ & Beta & $R^{2}$ & $p$ \\
\hline $\mathrm{Hb}$ level, $\mathrm{mmol} / \mathrm{L}$ & $-1322(-1596$ to -1048$)$ & -0.765 & 0.585 & $<0.001$ & $-986(-1309$ to -664$)$ & -0.571 & & $<0.001$ \\
\hline CoVar $\mathrm{r}_{5} \mathrm{SO}_{2}$, per $10 \%$ & $-1411(-1894$ to -928$)$ & -0.583 & 0.340 & $<0.001$ & $-838(-1235$ to -441$)$ & -0.346 & $0.675^{\mathrm{b}}$ & $<0.001$ \\
\hline
\end{tabular}

$B$ unstandardized coefficient, $C l$ confidence interval, Beta standardized coefficient, $I$-FABP intestinal fatty acid-binding protein, $R B C T$ red blood cell transfusion, $\mathrm{Hb}$ hemoglobin, $r_{5} \mathrm{SO}_{2}$ splanchnic tissue oxygen saturation, CoVar coefficient of variation.

${ }^{a}$ All variables with $p<0.10$ were included in the multivariable model;

${ }^{\mathrm{b}} R^{2}$ of the multivariable model.

in the presence of a PDA and mechanical ventilation between cases and controls, both factors did not affect the associations between $\mathrm{Hb}$ level, splanchnic oxygenation, and I-FABP. Cases had a higher incidence of a PDA and they required mechanical ventilation more often than controls. Both the presence of a PDA and mechanical ventilation may have affected (mesenteric) perfusion. After adjusting for the presence of a PDA and for mechanical ventilation separately, we, however, found that the results did not change regarding the association between lower hemoglobin level and higher urinary I-FABP levels. We stress the fact that we did not identify any clinical variables, other than $\mathrm{Hb}$ level, related to this early onset of intestinal cell injury.

We demonstrated an association between both low $\mathrm{Hb}$ and its concomitant low $\mathrm{r}_{5} \mathrm{SO}_{2}$, and high I-FABP levels already before the first RBC transfusion. Moreover, the association between $\mathrm{Hb}$ level and I-FABP disappeared in non-anemic matched controls. These findings support the theory regarding transfusion-associated NEC, that it is more the anemia itself and less the following RBC transfusion that gives rise to intestinal cell injury. Finally, the results of the multivariable model strengthen this theory. Of all variables, decreasing $\mathrm{Hb}$ level had the strongest relation with the level of urinary I-FABP. Further studies are needed to confirm our results and possibly to develop an individualized critical $\mathrm{Hb}$ threshold causing intestinal injury. Despite the fact that the TOP and ETTNO trial did not find a difference in NEC incidence between higher and lower RBC transfusion thresholds, ${ }^{50,51}$ future randomized trials comparing RBC transfusion thresholds may also focus on the prevention of intestinal injury.

We recognize several limitations of our study. First, we were unable to match all original infants who received an $\mathrm{RBC}$ transfusion. Low GA and low BW of the transfused infants hampered us in four cases to match those infants to appropriate controls. We, however, collected a rather complete dataset of prospectively collected data. Furthermore, the study design offers the possibility to disentangle the effect of anemia from the effect of RBC transfusions. Second, we relied on I-FABP as a biomarker for intestinal injury, being the best available at present for the prediction and extent of NEC, ${ }^{28-30,52}$ and with additional value as a biomarker for subtle intestinal injury, ${ }^{36,37,53}$ although the latter has been studied less often. Third, we performed our study in a single center. This did, however, allow us to enroll a homogenous study population with minimal variations in NICU practices, sample collection, and laboratory procedures. Finally, we included a relatively small sample size which hampered us from relating our results to the development of NEC. Finally, we did not correct for the potential effect of feeding.

\section{CONCLUSION}

In conclusion, this study demonstrated that low hemoglobin levels, low splanchnic oxygen saturation, and a diminished variability of splanchnic oxygen saturation in anemic preterm infants prior to RBC transfusion were associated with intestinal injury, reflected in increased levels of urinary I-FABP. This may entail an important hypoxic intestinal hit towards the development of NEC.

\section{REFERENCES}

1. Aher, S., Malwatkar, K. \& Kadam, S. Neonatal anemia. Semin. Fetal Neonatal Med. 13, 239-247 (2008).

2. Colombatti, R., Sainati, L. \& Trevisanuto, D. Anemia and transfusion in the neonate. Semin. Fetal Neonatal Med. 21, 2-9 (2016).

3. Blank, J. P. et al. The role of RBC transfusion in the premature infant. Am. J. Dis. Child. 138, 831-833 (1984).

4. Van Hoften, J. C. R., Verhagen, E. A., Keating, P., ter Horst, H. J. \& Bos, A. F. Cerebral tissue oxygen saturation and extraction in preterm infants before and after blood transfusion. Arch. Dis. Child. Fetal Neonatal Ed. 95, F352-F358 (2010).

5. Keir, A. K. et al. Temporal changes in blood product usage in preterm neonates born at less than 30 weeks' gestation in Canada. Transfusion 55, 1340-1346 (2015).

6. Singh, R. et al. Association of necrotizing enterocolitis with anemia and packed red blood cell transfusions in preterm infants. J. Perinatol. 31, 176-182 (2011).

7. Patel, R. M. et al. Association of red blood cell transfusions, anemia, and necrotizing enterocolitis in very low-birth-weight infants. JAMA 315, 889-897 (2016).

8. Ozcan, B., Aydemir, O., Isik, D. U., Bas, A. Y. \& Demirel, N. Severe anemia is associated with intestinal injury in preterm neonates. Am. J. Perinatol. 37 603-606 (2020).

9. Arthur, C. M. et al. Anemia induces gut inflammation and injury in an animal model of preterm infants. Transfusion 59, 1233-1245 (2019).

10. MohanKumar, K. et al. Severe neonatal anemia increases intestinal permeability by disrupting epithelial adherens junctions. Am. J. Physiol. Gastrointest. Liver Physiol. 318, G705-G716 (2020).

11. Christensen, R. D. et al. Is "transfusion-associated necrotizing enterocolitis" an authentic pathogenetic entity? Transfusion 50, 1106-1112 (2010).

12. Josephson, C. D. et al. Do red cell transfusions increase the risk of necrotizing enterocolitis in premature infants? J. Pediatr. 157, 972-978 (2010).

13. Paul, D. A. et al. Increased odds of necrotizing enterocolitis after transfusion of red blood cells in premature infants. Pediatrics 127, 635-641 (2011).

14. Blau, J. et al. Transfusion-related acute gut injury: necrotizing enterocolitis in very low birth weight neonates after packed red blood cell transfusion. J. Pediatr. 158, 403-409 (2011).

15. Mohamed, A. \& Shah, P. S. Transfusion associated necrotizing enterocolitis: a meta-analysis of observational data. Pediatrics 129, 529-540 (2012).

16. La Gamma, E. F. \& Blau, J. Transfusion-related acute gut injury: feeding, flora, flow, and barrier defense. Semin. Perinatol. 36, 294-305 (2012).

17. Stritzke, A. I., Smyth, J., Synnes, A., Lee, S. K. \& Shah, P. S. Transfusion-associated necrotizing enterocolitis in neonates. Arch. Dis. Child. Fetal Neonatal Ed. 98, F10-F14 (2013).

18. Cunningham, K. E., Okolo, F. C., Baker, R., Mollen, K. P. \& Good, M. Red blood cell transfusion in premature infants leads to worse necrotizing enterocolitis outcomes. J. Surg. Res. 213, 158-165 (2017).

19. Kirpalani, H. \& Zupancic, J. A. Do transfusions cause necrotizing enterocolitis? The complementary role of randomized trials and observational studies. Semin. Perinatol. 36, 269-276 (2012).

20. Wallenstein, M. B. et al. Red blood cell transfusion is not associated with necrotizing enterocolitis: a review of consecutive transfusions in a tertiary neonatal intensive care unit. J. Pediatr. 165, 678-682 (2014).

21. Sharma, R. et al. Packed red blood cell transfusion is not associated with increased risk of necrotizing enterocolitis in premature infants. J. Perinatol. 34, 858-862 (2014). 
22. Hyung, N. et al. The relationship of red blood cell transfusion to intestinal mucosal injury in premature infants. J. Pediatr. Surg. 52, 1152-1155 (2017).

23. Le, V. T., Klebanoff, M. A., Talavera, M. M. \& Slaughter, J. L. Transient effects of transfusion and feeding advances (volumetric and caloric) on necrotizing enterocolitis development: a case-crossover study. PLoS ONE 12, e0179724 (2017).

24. Hay, S., Zupancic, J. A. F., Flannery, D. D., Kirpalani, H. \& Dukhovny, D. Should we believe in transfusion-associated enterocolitis? Applying a GRADE to the literature. Semin. Perinatol. 41, 80-91 (2017).

25. AlFaleh, K. et al. Association of packed red blood cell transfusion and necrotizing enterocolitis in very low birth weight infants. J. Neonatal Perinat. Med. 7, 193-198 (2014).

26. Sood, B. G., Rambhatla, A., Thomas, R. \& Chen, X. Decreased hazard of necrotizing enterocolitis in preterm neonates receiving red cell transfusions. J. Matern. Fetal Neonatal Med. 29, 737-744 (2016).

27. Mintzer, J. P. \& Moore, J. E. Regional tissue oxygenation monitoring in the neonatal intensive care unit: evidence for clinical strategies and future directions. Pediatr. Res. 86, 296-304 (2019).

28. Schat, T. E. et al. The relation between splanchnic ischaemia and intestinal damage in necrotizing enterocolitis. Arch. Dis. Child. Fetal Neonatal Ed. 101, F533-F539 (2016).

29. Schurink, M. et al. Intestinal fatty acid-binding protein in neonates with imminent necrotizing enterocolitis. Neonatology 106, 49-54 (2014).

30. Schurink, M. et al. Intestinal fatty acid-binding protein as a diagnostic marker for complicated and uncomplicated necrotizing enterocolitis: a prospective cohort study. PLoS ONE 10, e0121336 (2015).

31. Von Lindern, J. S., Brand, A. \& Lopriore, E. Revision of the guideline 'Blood transfusion': for the newborn. Ned. Tijdschr. Geneeskd. 156, A4795 (2012).

32. Naulaers, G. et al. Use of tissue oxygenation index and fractional tissue oxygen extraction as non-invasive parameters for cerebral oxygenation. A validation study in piglets. Neonatology 92, 120-126 (2007).

33. Mintzer, J. P., Parvez, B., Chelala, M., Alpan, G. \& LaGamma, E. F. Quiescent variability of cerebral, renal, and splanchnic regional tissue oxygenation in very low birth weight neonates. J. Neonatal Perinat. Med. 7, 199-206 (2014).

34. Diez, S. et al. Clinical characteristics of necrotizing enterocolitis in preterm patients with and without persistent ductus arteriosus and in patients with congenital heart disease. Front. Pediatr. 8, 257 (2020).

35. Cavalcanti, L. S. et al. Serial Doppler velocimetry of mesenteric and portal flow in very-low-birth-weight preterm neonates with and without patent ductus arteriosus. Pediatr. Radiol. 50, 1107-1114 (2020).

36. Reisinger, K. W. et al. Intestinal fatty acid-binding protein: a possible marker for gut maturation. Pediatr. Res. 76, 261-268 (2014).

37. Elnady, H. G. et al. Prediction of gut wall integrity loss in viral gastroenteritis by non-invasive marker. Open Access Maced. J. Med. Sci. 3, 37-45 (2015).

38. Kuik, S. J. et al. Predicting intestinal recovery after necrotizing enterocolitis in preterm infants. Pediatr. Res. 87, 903-909 (2020).

39. Heida, F. H. et al. A necrotizing enterocolitis-associated gut microbiotia is present in the meconium: results of a prospective study. Clin. Infect. Dis. 62, 863-870 (2016).

40. Schat, T. E. et al. Early cerebral and intestinal oxygenation in the risk assessment of necrotizing enterocolitis in preterm infants. Early Hum. Dev. 131, 75-80 (2019).

41. Martini, S. \& Corvaglia, L. Splanchnic NIRS monitoring in neonatal care: rationale, current applications and future perspectives. J. Perinatol. 38, 431-443 (2018).

42. Koehler, R. C., Traystman, R. J. \& Jones, M. D. Jr. Regional blood flow and O2 transport during hypoxic and CO hypoxia in neonatal and adult sheep. Am. J. Physiol. 248, H118-H124 (1985).

43. El-Dib, M., Narang, S., Lee, E., Massaro, A. N. \& Aly, H. Red blood cell transfusion, feeding, and necrotizing enterocolitis in preterm infants. J. Perinatol. 31, 183-187 (2011).

44. Fairchild, K. D. Predictive monitoring for early detection of sepsis in neonatal ICU patients. Curr. Opin. Pediatr. 25, 172-179 (2013).

45. Javorka, K. et al. Heart rate variability in newborns. Physiol. Res. 66, S203-S214 (2017).

46. Cortez, J. et al. Noninvasive evaluation of splanchnic tissue oxygenation using near-infrared spectroscopy in preterm neonates. J. Matern. Fetal Neonatal Med. 24, 574-582 (2011).

47. Schat, T. E. et al. Near-infrared spectroscopy to predict the course of necrotizing enterocolitis. PLOS ONE 11, e0154710 (2016).

48. Kalteren, W. S. et al. Red blood cell transfusions affect intestinal and cerebral oxygenation differently in preterm infants with and without subsequent necrotizing enterocolitis. Am. J. Perinatol. 35, 1031-1037 (2018).
49. Van der Heide, M., Hulscher, J. B. F., Bos, A. F. \& Kooi, E. M. W. Near-infrared spectroscopy as a diagnostic tool for necrotizing enterocolitis in preterm infants. Pediatr. Res. 90, 148-155 (2021).

50. Franz, A. R. et al. Effects of liberal vs restrictive transfusion thresholds on survival and neurocognitive outcomes in extremely low-birth-weight infants: the ETTNO randomized clinical trial. JAMA 324, 560-570 (2020).

51. Kirpalani, H. et al. Higher or lower hemoglobin transfusion thresholds for preterm infants. N. Engl. J. Med. 383, 2639-2651 (2020).

52. Heida, F. H. et al. Intestinal fatty acid-binding protein levels in necrotizing enterocolitis correlate with extent of necrotic bowel: results from a multicenter study. J. Pediatr. Surg. 50, 1115-1118 (2015).

53. Kokesova, A. et al. The intestinal fatty acid-binding protein as a marker for intestinal damage in gastroschisis. PLoS ONE 14, e0210797 (2019).

\section{ACKNOWLEDGEMENTS}

We greatly acknowledge the patients and their parents for participating in the POCKET-study and/or STEP-trial. Furthermore, we acknowledge the nurses, medical staff, and students of the NICU at the Beatrix Children's Hospital in Groningen for helping to collect data. We also thank Ms. A.J. Olthuis, Ms. D.H. Baptist, and Ms. H.A. Bouma for their help with patient inclusion and data collection. This study was part of the research program of the Graduate School of Medical Sciences, Research Institute SHARE, University of Groningen.

\section{AUTHOR CONTRIBUTIONS}

W.S.K. conceptualized and designed the study, collected data, carried out and interpreted the analyses, drafted the initial manuscript, and critically reviewed and revised the manuscript for important intellectual content. A.F.B. conceptualized and designed the study, supervised and interpreted the analyses, and critically reviewed and revised the manuscript for important intellectual content. W.v.O. performed laboratory analyses, and critically reviewed, and revised the manuscript for important intellectual content. J.B.F.H. conceptualized and designed the study, and critically reviewed, and revised the manuscript for important intellectual content. E.M.W.K. conceptualized and designed the study, coordinated and supervised data collection, supervised and interpreted the analyses, and critically reviewed and revised the manuscript for important intellectual content. All authors approved the final manuscript as submitted and agree to be accountable for all aspects of the work.

\section{FUNDING}

Ms. W.S. Kalteren received a grant from the Tekke Huizinga Fonds, Groningen, the Netherlands. Ms. W.S. Kalteren also received financial support from the Junior Scientific Master Class of the University of Groningen. The funders had no role in study design, data collection and analysis, decision to publish, or preparation of the manuscript.

\section{COMPETING INTERESTS}

The authors declare no competing interests.

\section{CONSENT STATEMENT}

All parents or guardians of neonates provided written informed consent.

\section{ADDITIONAL INFORMATION}

Correspondence and requests for materials should be addressed to Willemien $\mathrm{S}$. Kalteren.

Reprints and permission information is available at http://www.nature.com/ reprints

Publisher's note Springer Nature remains neutral with regard to jurisdictional claims in published maps and institutional affiliations. 